[Article]

www.whxb.pku.edu.cn

\title{
牛血清白蛋白的光损伤和光氧化机理
}

程伶俐赵萍王玫朱慧朱融融孙晓宇 汪世龙
(同济大学生命科学与技术学院, 上海 200092)

\begin{abstract}
摘要：运用激光闪光光解瞬态吸收技术, 在 $266 \mathrm{~nm}$ 激光激励下, 研究了牛血清白蛋白(BSA)光损伤和被 $\mathrm{SO}_{4}^{-*}$ 单电子氧化的反应机理, 表征了反应过程中生成的自由基. 结果表明, 在 $266 \mathrm{~nm}$ 激光照射下, BSA可同时发生光 电离和光激发, 生成色氨酸阳离子自由基 $\left(\mathrm{Trp} / \mathrm{NH}^{+\bullet}\right)$, 由 $\mathrm{Trp} / \mathrm{NH}^{+\bullet}$ 快速脱质子形成的色氨酸中性自由基 $\left(\mathrm{Trp} / \mathrm{N}^{\bullet}\right)$ 及色氨酸三重激发态 $\left({ }^{3} \mathrm{Trp}^{*}\right),{ }^{3} \mathrm{Tr}{ }^{*}$ 再与酪氨酸 $(\mathrm{Tyr})$ 发生分子内电子转移生成酪氨酸中性自由基 $\left(\mathrm{Tyr} / \mathrm{O}^{\bullet}\right)$. 在 $\mathrm{SO}_{4}^{-}$ 单电子氧化的反应中, 借助减谱技术, 求得BSA中Tyr和色氨酸(Trp)自由基的表观生成速率常数, 但未发现分子 内电子转移现象, 阐明了 $\mathrm{SO}_{4}^{-}$自由基是通过与 $\mathrm{BSA}$ 中的 $\mathrm{Tyr}$ 和Trp发生电子转移反应来氧化 $\mathrm{BSA}^{-}$的, $\mathrm{SO}_{4}^{-\bullet}$ 氧化 $\mathrm{BSA}$ 的反应速率常数为 $1.51 \times 10^{10} \mathrm{~L} \cdot \mathrm{mol}^{-1} \cdot \mathrm{s}^{-1}$, 从而为进一步研究血清白蛋白的氧化还原代谢过程提供理论基础.
\end{abstract}

关键词: 牛血清白蛋白; 光电离; 光激发; 电子转移; 单电子氧化

中图分类号: $\mathrm{O} 644$

\section{Photodamage and Photooxidation Mechanisms of Bovine Serum Albumin}

\author{
CHENG Ling-Li ZHAO Ping WANG Mei ZHU Hui \\ ZHU Rong-Rong SUN Xiao-Yu WANG Shi-Long* \\ (School of Life Science and Technology, Tongji University, Shanghai 200092, P. R. China)
}

\begin{abstract}
Photodamage and photooxidation mechanisms of bovine serum albumin (BSA) as induced by UV radiation and one-electron oxidation by $\mathrm{SO}_{4}^{-\bullet}$ were investigated by $266 \mathrm{~nm}$ laser flash photolysis. BSA can be photoionized and photoexcited by $266 \mathrm{~nm}$ photons to give tryptophan $(\operatorname{Trp}) / \mathrm{NH}^{+}, \operatorname{Trp} / \mathrm{N}^{\bullet}$ resulting from the rapid deprotonation of $\operatorname{Trp} /$ $\mathrm{NH}^{+\bullet}$, and ${ }^{3} \mathrm{Trp}^{*}$. Through intermolecular electron transfer between ${ }^{3} \mathrm{Trp}^{*}$ and tyrosine (Tyr), Tyr/O ${ }^{\bullet}$ was produced. In one-electron oxidation of BSA by $\mathrm{SO}_{4}^{-}$the apparent set up rate constants of Tyr and Trp radicals were calculated but intermolecular electron transfer was not observed. We propose that $\mathrm{SO}_{4}^{-\bullet}$ oxidizes BSA by electron transfer to the Tyr and Trp of BSA with a rate constant of $1.51 \times 10^{10} \mathrm{~L} \cdot \mathrm{mol}^{-1} \cdot \mathrm{s}^{-1}$. This research provides an introductory theory to enable further study on the redox metabolic process of BSA.
\end{abstract}

Key Words: Bovine serum albumin; Photo-ionization; Photo-excitation; Electron transfer; One-electron oxidization

蛋白质是生物体中一类重要的大分子, 对于维 持生命是十分重要和必不可少的. 血清白蛋白是哺 乳动物血浆中含量最丰富的蛋白质, 它能够储存和 转运众多内源性和外源性物质. 由于血清白蛋白在 生理上的重要性以及易于分离、提纯而常被用作模
型球蛋白 ${ }^{[1-4]}$. 牛血清白蛋白(BSA)由于其重要的生 物化学和生物物理学意义, 在过去的 40 年内被广泛 地研究 ${ }^{[5]}$. BSA 是一种广泛应用的球蛋白, 它的分子 结构中含有 583 个氨基酸残基, 其中有 78 个氨基酸 容易被氧化 $(2$ 个色氨酸、 4 个甲硫氨酸、 17 个组氨

Received: July 25, 2008; Revised: September 30, 2008; Published on Web: November 12, 2008.

*Corresponding author. Email: ws1@mail.tongji.edu.cn; Tel: +8621-65982595.

国家自然科学基金(30570376, 50673078)、上海市基础重点项目(06JC14068)及上海市教委科技创新项目(08DZ21)资助

C Editorial office of Acta Physico-Chimica Sinica 
酸、20 个酪氨酸和 35 个半胱氨酸 $)^{[6]}$, 作为动物体内 重要的转运蛋白, BSA 参与了动物体内一系列重要 的氧化还原反应. 在光生物学和辐射生物学中, 光及 电离辐射与氨基酸、肽链及蛋白质相互作用是近年 来生命科学研究的热门课题, 由于这些研究均涉及 自由基和激发态过程, 与生物体内的氧化还原反应, 酶催化反应及生物体对辐射(包括紫外光)的保护和 敏化作用有着极其密切的关系 ${ }^{[7]}$, 所以探讨 BSA 的 光损伤和光氧化反应机理就显得非常必要.

由于生命体系的原初反应过程都是微观动态过 程, 本研究采用纳秒激光光解技术研究了 BSA 的光 损伤和 $\mathrm{SO}_{4}^{-}$- 自由基氧化 $\mathrm{BSA}$ 的反应, 初步阐述了 BSA 在水溶液中的光损伤和光氧化反应机理, 从而 为进一步研究血清白蛋白的氧化还原代谢过程提供 理论基础.

\section{1 实验部分}

\section{1 实验试剂}

牛血清白蛋白(BSA, 99\%, Sigma公司), 未经进 一步纯化直接使用. 过硫酸钾 $\left(\mathrm{K}_{2} \mathrm{~S}_{2} \mathrm{O}_{8}\right)$ (分析纯, 国药 集团化学试剂有限公司), 重结晶后使用, 其它试剂 均为分析纯. 实验体系均为磷酸盐缓冲液 $(\mathrm{pH}=7) ; \mathrm{N}_{2}$ 、 $\mathrm{N}_{2} \mathrm{O}$ 和 $\mathrm{O}_{2}$ 为 $99.99 \%$ 的高纯气体. 除非特殊说明, 本 实验所用溶液均用 Millipore 纯水配制, 并且保存在 暗处, 尽量避免光照. 溶液根据需要, 分别在实验前 用高纯气体鼓泡 $20 \mathrm{~min}$ 处理研究样品, 所有实验均 在室温下进行.

\section{2 实验装置}

研究在同济大学生命科学与技术学院的纳秒级 激光光解装置上进行 ${ }^{[8,9]}$, 采用YAG固体激光器为激 励光源, 脉宽为 3-6 ns, 输出激光波长为 $266 \mathrm{~nm}$ 时, 单脉冲最大能量为 $60 \mathrm{~mJ}$, 氙灯作为全光谱分析检 测光源, 与激光光路垂直, 分析光经透镜系统汇聚于 $1 \mathrm{~cm}$ 石英样品池, 经单色仪分光后由 $\mathrm{R} 955$ 光电倍 增管转化为电信号, 为安捷伦 $1 \mathrm{G}$ 高速数字示波器 记录, 数据转至计算机后用自编软件进行分析处理. 可控流量通气仪为本实验室研制; 所用仪器还有紫 外-可见分光光度仪 (Cary 50 Probe, VARIAN, 美 国)、电子分析天平(AL204, Mettler Toledo, 瑞士)、pH 计(DELTA 320, Mettler Toledo, 瑞士)、超声清洗仪 (DL-360D, 上海之信仪器有限公司)、恒温干燥箱 (DHG-9140A, 上海精宏实验设备有限公司)及超纯 水器(Milli-Q, Millipore, 美国).

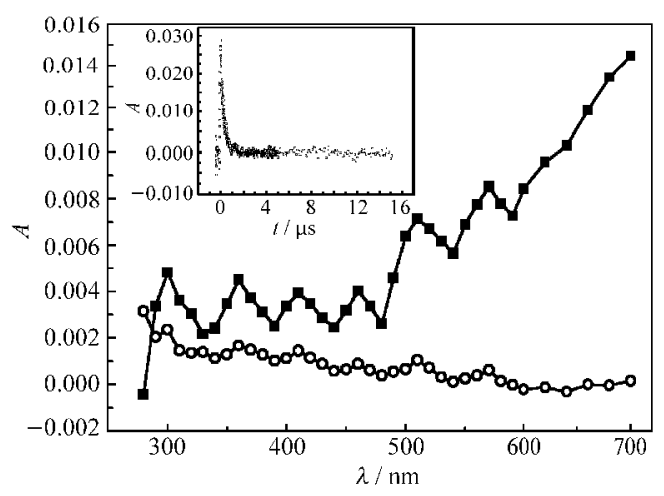

图 1 BSA 激光光解后不同时刻的瞬态吸收光谱

Fig.1 Transient absorption spectra of BSA

The spectra were recorded at $0.1 \mu \mathrm{s}(\mathbf{\square}), 5 \mu \mathrm{s}(\bigcirc)$ following $266 \mathrm{~nm}$ laser pulse excitation of $\mathrm{N}_{2}$-saturated aqueous solution containing $7.55 \times 10^{-6} \mathrm{~mol} \cdot \mathrm{L}^{-1} \mathrm{BSA}$. Inset shows the transient absorption curve observed at $680 \mathrm{~nm}$.

\section{2 结果与讨论}

\section{$2.1266 \mathrm{~nm}$ 激光作用下 BSA 光损伤机理研究}

配制 $7.55 \times 10^{-6} \mathrm{~mol} \cdot \mathrm{L}^{-1}$ 氮气饱和的 BSA 中性 水溶液, $266 \mathrm{~nm}$ 激光光解后得到图 1 所示不同时刻 的瞬态吸收谱. 从图 1 可以看出, BSA 水溶液在 266 $\mathrm{nm}$ 的激光作用后, 在 $0.1 \mu \mathrm{s}$ 时, 在 500-750 nm 范 围内出现瞬态吸收, 通人水合电子清除剂 $\mathrm{N}_{2} \mathrm{O}$ 后该 区域的瞬态吸收几乎消失(图 2), 具有水合电子的瞬 态吸收特征, 因此可判定这一吸收为水合电子的贡 献. 根据水合电子的存在, 可以初步判定 BSA 在 $266 \mathrm{~nm}$ 激光的作用下发生了光电离 ${ }^{[10]}$.

分别对比在 $\mathrm{N}_{2} 、 \mathrm{~N}_{2} \mathrm{O}$ 和 $\mathrm{O}_{2}$ 饱和条件下, 同一时 刻 BSA 中性水溶液的瞬态吸收光谱(图 2), 在 $\mathrm{N}_{2}$ 和

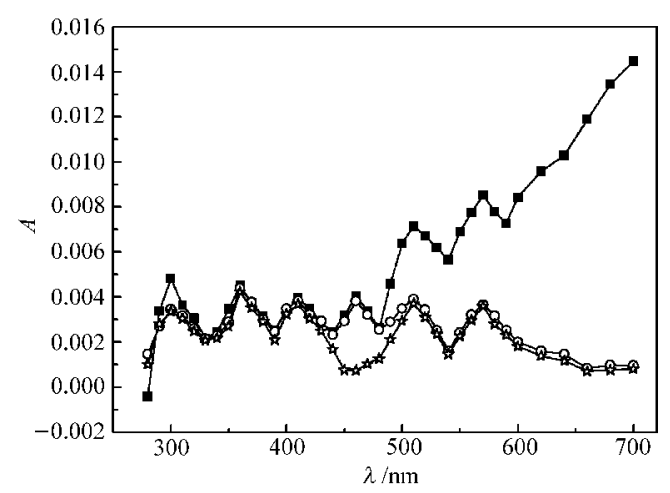

图 2 不同气氛下 BSA 激光光解后的瞬态吸收光谱

Fig.2 Transient absorption spectra of BSA recorded at different saturated solutions

The spectra were recorded at $0.1 \mu$ s following $266 \mathrm{~nm}$ laser pulse excitation of $\mathrm{N}_{2}$-saturated $(\mathbf{\square}), \mathrm{N}_{2} \mathrm{O}$-saturated $(\mathrm{O})$, and $\mathrm{O}_{2}$-saturated (纤) aqueous solution containing $7.55 \times 10^{-6} \mathrm{~mol} \cdot \mathrm{L}^{-1} \mathrm{BSA}$. 
$\mathrm{N}_{2} \mathrm{O}$ 饱和条件下, 均可以观察到 $360 、 410 、 460 、 510$ 和 $570 \mathrm{~nm}$ 的特征吸收峰, 而在 $\mathrm{O}_{2}$ 饱和条件下, 460 $\mathrm{nm}$ 处的吸收峰消失. 由于 $\mathrm{O}_{2}$ 是激发三重态的猝灭 剂, 意味着 $460 \mathrm{~nm}$ 处可能是激发三重态的贡献. Bensasson等 ${ }^{[11}$ 曾经报道过, 蛋白质中的芳香氨基酸, 由于其氧化电位是氨基酸中最低的, 对 UV 光和氧 化剂敏感, 因此是蛋白质光氧化损伤的重要位置. 在 蛋白质的化学和放射性损伤过程中, 常常出现色氨 酸吲哚自由基 $\left(\mathrm{TrpN}^{\bullet}\right)$ 和酪氨酸酚氧自由基( $\left.\mathrm{TyrO}^{\bullet}\right)$. 根据文献[12-15]报道, ${ }^{3} \operatorname{Trp} *$ 的特征吸收在 $460 \mathrm{~nm}$ 处, 而 $\mathrm{Trp} / \mathrm{NH}^{+\bullet}$ 的特征吸收在约 350 和 $560 \mathrm{~nm}$ 处, $\mathrm{TrpN}^{\bullet}$ 和 $\mathrm{TyrO}{ }^{\bullet}$ 的特征吸收分别位于约 520 和 410 $\mathrm{nm}$ 处. 故上述 BSA 中性水溶液瞬态吸收谱中 360 和 $570 \mathrm{~nm}$ 处的特征吸收峰可归属为 $\mathrm{Trp} / \mathrm{NH}^{+\bullet}$ 的贡 献, $510 \mathrm{~nm}$ 处的可归属为 $\mathrm{TrpN} \mathrm{N}^{\bullet}$ 的贡献, $410 \mathrm{~nm}$ 处的 可归属为 $\mathrm{TyrO}{ }^{\circ}$ 的贡献, $460 \mathrm{~nm}$ 处的归属为 ${ }^{3} \mathrm{Trp}{ }^{*}$ 的 贡献.

对比氮气饱和条件下, 460 和 $410 \mathrm{~nm}$ 处时间衰 减谱图(图3), 观察到伴随着 $460 \mathrm{~nm}$ 处 ${ }^{3} \mathrm{Trp}$ *的衰减, $410 \mathrm{~nm}$ 处 $\mathrm{TyrO}$ 呈现一个迅速生成, 然后缓慢衰减 的过程. 故初步可推断体系经历了两个过程: 首先, Trp 残基被光激发生成 ${ }^{3} \mathrm{Trp} *$, 随后 ${ }^{3} \mathrm{Trp}$ * 通过分子 内电子转移氧化 $\mathrm{Tyr}$ 残基, 生成 $\mathrm{TyrOH}^{+}$, 其 $\mathrm{p} K_{\mathrm{a}}$ 值 (弱酸性或弱碱性物质在 $50 \%$ 解离时溶液的 $\mathrm{pH}$ 值) 为 -1 , 在此体系中迅速脱质子生成 $\mathrm{TyrO}^{\text {•[16]. 其原因可 }}$ 能是, $\operatorname{Trp}$ 在 $266 \mathrm{~nm}$ 的摩尔消光系数比 $\mathrm{Tyr}$ 大近 10 倍, 在此体系中, 激光能量主要被 $\operatorname{Tr}$ 吸收, 光解生成

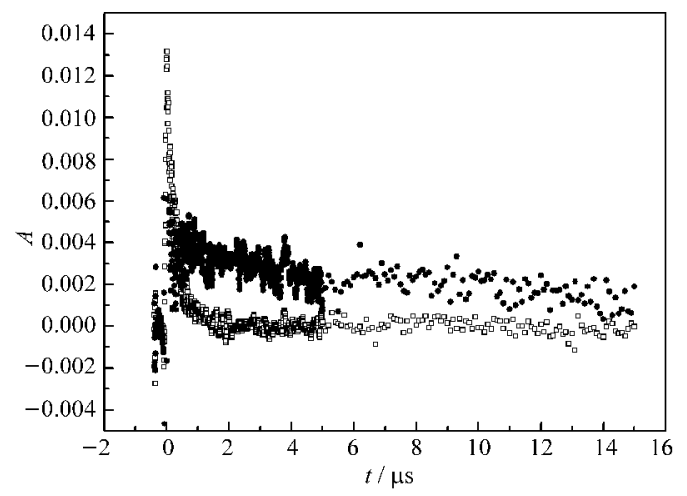

图 3 BSA 激光光解后在 $460 \mathrm{~nm}(\square)$ 和 $410 \mathrm{~nm}(\bullet)$ 记录的时间衰减谱曲线

Fig.3 Transient absorption curves of BSA at $460 \mathrm{~nm}(\square)$ and $410 \mathrm{~nm}(\bullet)$

$\mathrm{N}_{2}$-saturated aqueous solution containing $7.55 \times 10^{-6} \mathrm{~mol} \cdot \mathrm{L}^{-1}$ BSA excited by $266 \mathrm{~nm}$ laser pulse.
$\operatorname{TrpNH}^{+\bullet}$ 和 ${ }^{3} \operatorname{Trp}{ }^{*} . \operatorname{Trp}$ 的 $\mathrm{p} K_{\mathrm{a}}$ 值为 4.3 , 在此体系中会迅 速脱质子形成 $\operatorname{TrpN}{ }^{\bullet}$, 而由于 $\mathrm{Tyr}$ 的氧化电位比 $\operatorname{Trp}$ 低(Trp 1.05 V, Tyr 0.94 V(vs NHE) $)^{[17]}$, 故 Tyr 可以被 $\operatorname{Trp}$ 所氧化, 且 ${ }^{3} \operatorname{Trp}{ }^{*}$ 的氧化性大于 $\operatorname{TrpNH}{ }^{+}$和 $T r p N^{\bullet}$, 所以推测是由 ${ }^{3} \mathrm{Trp}$ *通过分子内电子转移氧化 $\mathrm{Tyr}$ 残基, 最终生成 $\mathrm{TyrO}^{\circ}$.

\section{$2.2266 \mathrm{~nm}$ 激光作用下 $\mathrm{SO}_{4}^{-*}$ 自由基氧化 BSA 的 机理研究}

实验体系中 $\mathrm{K}_{2} \mathrm{~S}_{2} \mathrm{O}_{8}$ 与 $\mathrm{BSA}$ 的浓度配比是将二 者的 $266 \mathrm{~nm}$ 吸光度之比保持在 100 以上, 这样可

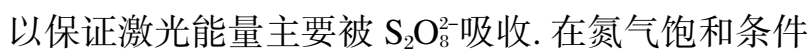
下, $0.05 \mathrm{~mol} \cdot \mathrm{L}^{-1}$ 过硫酸钾中性水溶液经 $266 \mathrm{~nm}$ 激 光激发后得到的 $0.1 \mu \mathrm{s}$ 瞬态吸收谱具有 300-570 nm 处的宽吸收峰, 其中特征吸收位于 $460 \mathrm{~nm}$ 处(见图 4 插图), 此谱与文献中得到的过硫酸钾瞬态吸收谱完 全吻合, 可将其归为 $\mathrm{SO}_{4}^{-\bullet}$ 自由基的瞬态吸收谱 ${ }^{[18]}$. 当 含有 $7.55 \times 10^{-6} \mathrm{~mol} \cdot \mathrm{L}^{-1} \mathrm{BSA}$ 和 $0.05 \mathrm{~mol} \cdot \mathrm{L}^{-1}$ 过硫酸 钾中性水溶液经 $266 \mathrm{~nm}$ 激光激发后, $10 \mu \mathrm{s}$ 时得到 的瞬态吸收谱如图 4 所示. 激光光解后, 很快可以观 察到 $\mathrm{SO}_{4}^{-\bullet}$ 自由基的特征吸收峰, 随着 $\mathrm{SO}_{4}^{-\bullet}$ 自由基的 衰减, 含 BSA 的二元体系在 $350 、 410 、 520$ 和 560 $\mathrm{nm}$ 处有新的吸收, 说明有新的瞬态物种生成, 由于 在此体系中激光能量基本都被过硫酸钾吸收, BSA 几乎不发生光电离, 所以新的瞬态物种是由过硫酸 钾光电离产生的 $\mathrm{SO}_{4}^{-\bullet}$ 自由基氧化 BSA 产生的. 改 变 $\mathrm{BSA}$ 的浓度, 发现 $\mathrm{SO}_{4}^{-\bullet}$ 自由基的衰减随 $\mathrm{BSA}$ 浓 度增加而加快(图5), 进一步说明 $\mathrm{SO}_{4}^{-\bullet}$ 自由基确实与

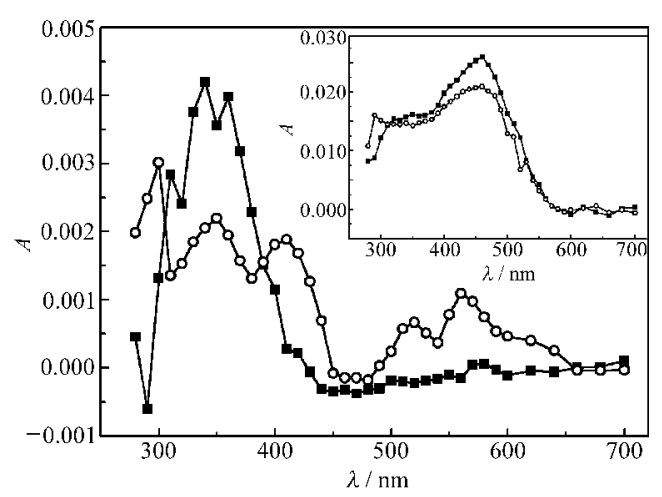

图 4 过硫酸钾在有 $(\bigcirc)$ 无 $(\boldsymbol{\square}) \mathrm{BSA}$ 条件下的瞬态吸收谱 Fig.4 Transient absorption spectra of $\mathrm{K}_{2} \mathrm{~S}_{2} \mathrm{O}_{8}$ without $(\square)$ or with $(\bigcirc)$ BSA

The spectra were recorded at $10 \mu$ s following $266 \mathrm{~nm}$ laser pulse excitation of $\mathrm{N}_{2}$-saturated aqueous solution containing $0.05 \mathrm{~mol} \cdot \mathrm{L}^{-1}$ $\mathrm{K}_{2} \mathrm{~S}_{2} \mathrm{O}_{8}$ and $7.55 \times 10^{-6} \mathrm{~mol} \cdot \mathrm{L}^{-1} \mathrm{BSA}(\mathrm{O})$ or only containing $0.05 \mathrm{~mol} \cdot \mathrm{L}^{-1}$ $\mathrm{K}_{2} \mathrm{~S}_{2} \mathrm{O}_{8}(\boldsymbol{\square})$. Inset shows the spectra recorded at $0.1 \mu$ s under the same condition. 


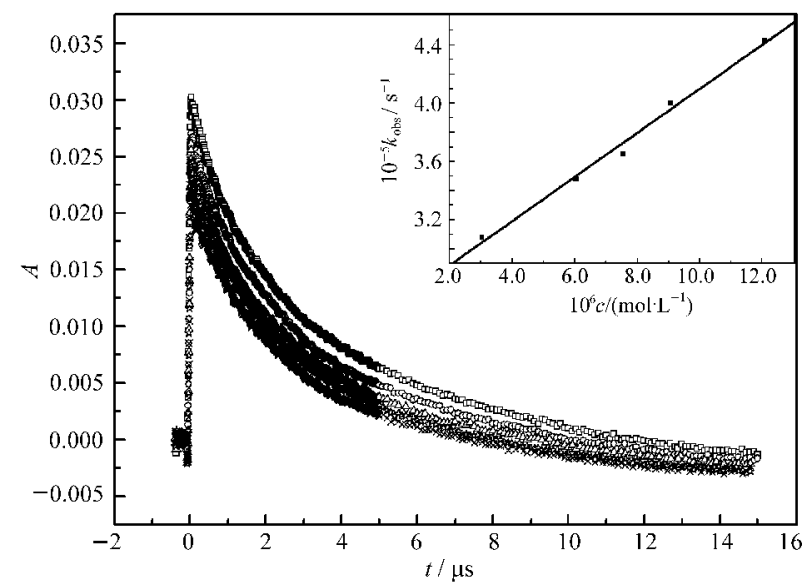

图 5 过硫酸钾在不同浓度 BSA 时 $460 \mathrm{~nm}$ 处的时间衰减 谱图

Fig.5 Transient absorption curves of $\mathrm{K}_{2} \mathrm{~S}_{2} \mathrm{O}_{8}$ with different concentrations of BSA at $460 \mathrm{~nm}$

$\mathrm{N}_{2}$-saturated aqueous solution containing $0.05 \mathrm{~mol} \cdot \mathrm{L}^{-1} \mathrm{~K}_{2} \mathrm{~S}_{2} \mathrm{O}_{8}$ with $3.02 \times 10^{-6}(\square), 6.04 \times 10^{-6}(\bigcirc), 7.55 \times 10^{-6}(\triangle), 9.06 \times 10^{-6}$ (论), or $12.08 \times 10^{-6}(\times) \mathrm{mol} \cdot \mathrm{L}^{-1} \mathrm{BSA}$ excited by $266 \mathrm{~nm}$ laser pulse. Inset shows the dependence of the $\mathrm{SO}_{4}^{-\bullet}$ decay rate constant $\left(k_{\mathrm{obs}}\right)$ with concentration of BSA.

BSA 发生了作用. 因此, 在研究的体系中, 初步判断 $10 \mu \mathrm{s}$ 时得到的瞬态吸收谱应该是 $\mathrm{SO}_{4}^{-\bullet}$ 自由基和氨 基酸自由基的叠加谱.

BSA中的 TrpNH和TyrOH残基提供电子给 $\mathrm{SO}_{4}^{-\bullet}$, 生成 $\mathrm{SO}_{4}^{2-}$ 和对应的氨基酸阳离子自由基 $\left(\mathrm{TrpNH}^{+\bullet}\right.$ 或

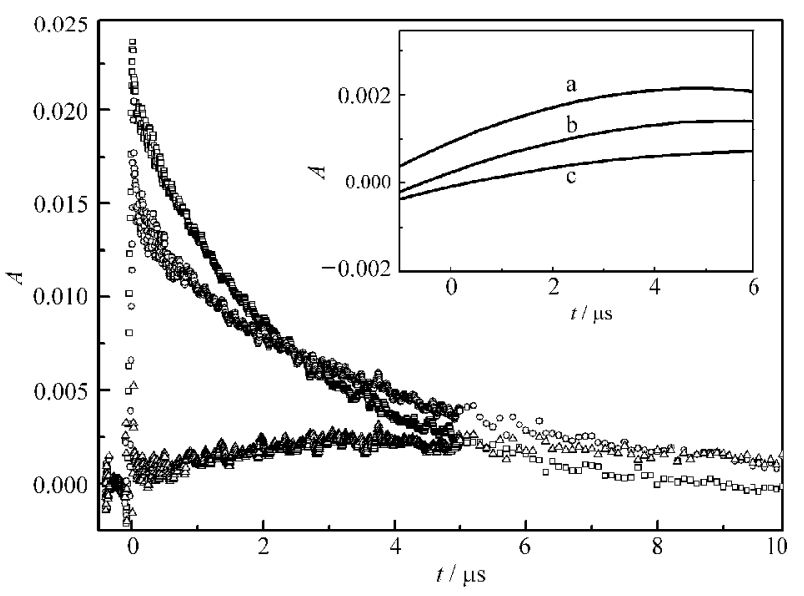

图 6 过硫酸钾和 BSA 激光光解后在 $350 \mathrm{~nm} \mathrm{( \bigcirc )、460}$ $\mathrm{nm}(\square)$ 和 350-460 nm ( $\triangle$ ) 处的时间衰减谱曲线

Fig.6 Transient absorption curves of $\mathrm{K}_{2} \mathrm{~S}_{2} \mathrm{O}_{8}$ and BSA at $350 \mathrm{~nm}(\bigcirc), 460 \mathrm{~nm}(\square)$, and 350-460 nm $(\triangle)$

$\mathrm{N}_{2}$-saturated aqueous solution containing $0.05 \mathrm{~mol} \cdot \mathrm{L}^{-1} \mathrm{~K}_{2} \mathrm{~S}_{2} \mathrm{O}_{8}$ and $7.55 \times 10^{-6} \mathrm{~mol} \cdot \mathrm{L}^{-1} \mathrm{BSA}$ excited by $266 \mathrm{~nm}$ laser pulse. $350-460 \mathrm{~nm}$ represents the set up and decay curve of $\mathrm{TrpNH}^{+}$obtained by substract $460 \mathrm{~nm}$ from $350 \mathrm{~nm}$. Inset shows the set up transient absorption curves observed at $520 \mathrm{~nm}$ (a), $410 \mathrm{~nm}$ (b), and $560 \mathrm{~nm}$ (c).
表 $1 \operatorname{TrpNH}^{+\bullet}$ 、TrpN`和 $\operatorname{TyrO} 0^{*}$ 表观生成速率常数 Table 1 Apparent set up rate constants of $\operatorname{TrpNH}^{+\bullet}$, TrpN' and TyrO•

\begin{tabular}{ccc}
\hline Transient product & Maximum absorption $(\mathrm{nm})$ & $10^{-5} k_{\text {obs }} / \mathrm{s}^{-1}$ \\
\hline $\operatorname{TrpNH}^{\bullet}$ & 350 & 2.78 \\
& 560 & 2.74 \\
$\mathrm{TrpN}^{\bullet}$ & 520 & 3.72 \\
$\mathrm{TyrO}^{\bullet}$ & 410 & 3.22 \\
\hline
\end{tabular}

$\left.\mathrm{TyrOH}^{+\bullet}\right)$. 根据前面对 $\mathrm{TrpNH} \mathrm{H}^{+\bullet}$ 和 $\mathrm{TyrOH}^{+\bullet}$ 特征吸收 峰的分析, 可将图4过硫酸钾和BSA二元体系中出现 的 350 和 $560 \mathrm{~nm}$ 的特征吸收峰归属为 $\mathrm{TrpNH}^{+\bullet}$ 的贡 献. 在本实验条件下 $(\mathrm{pH}=7), \operatorname{TrpNH}^{+\bullet}\left(\mathrm{p} K_{\mathrm{a}}=4.3\right)$ 和 $\mathrm{TyrOH}^{+\bullet}\left(\mathrm{p} K_{\mathrm{a}}=-1\right)$ 会脱质子, 转变为中性自由基 $\mathrm{TrpN}^{\bullet}$ 和 $\mathrm{TyrO}{ }^{\bullet}{ }^{[13,16]}$. 结合前面对 $\mathrm{TrpN}{ }^{\bullet}$ 和 $\mathrm{TyrO} \bullet$ 特征吸收谱 的分析, 可将图4过硫酸钾和BSA二元体系中出现 的 520 和 $410 \mathrm{~nm}$ 的特征吸收峰分别归属为 $\operatorname{TrpN} \mathrm{N}^{\bullet}$ 和 TyrO的贡献.

但由于氨基酸自由基的吸收和 $\mathrm{SO}_{4}^{-\bullet}$ 的吸收发 生重叠, 很难直接观察到氨基酸自由基的生成. 故采 用减谱技术 ${ }^{[19]}$, 扣除 $\mathrm{SO}_{4}^{-\bullet}$ 瞬态吸收的贡献, 得到了 $\mathrm{TrpNH}^{+}(350$ 和 $560 \mathrm{~nm}) 、 \mathrm{TrpN}^{\bullet}(520 \mathrm{~nm})$ 和 $\mathrm{TyrO}^{\bullet}$ (410 nm)的净生成曲线(图 6), 分别求得其表观生成 速率常数如表 1 所示. 在此体系中, 未观察到分子内 电子转移现象, 其可能的原因是在此体系中, 光子能 量主要被 $\mathrm{K}_{2} \mathrm{~S}_{2} \mathrm{O}_{8}$ 吸收, 生成强氧化剂 $\mathrm{SO}_{4}^{-\bullet}$, 故 Trp 不 可能发生光激发产生 ${ }^{3} \mathrm{Trp}^{*}$, 因此不能观察到上述的 分子内电子转移现象.

根据以上分析, 推断 $\mathrm{SO}_{4}^{-\bullet}$ 与 $\mathrm{BSA}$ 之间可能发 生了如下反应:

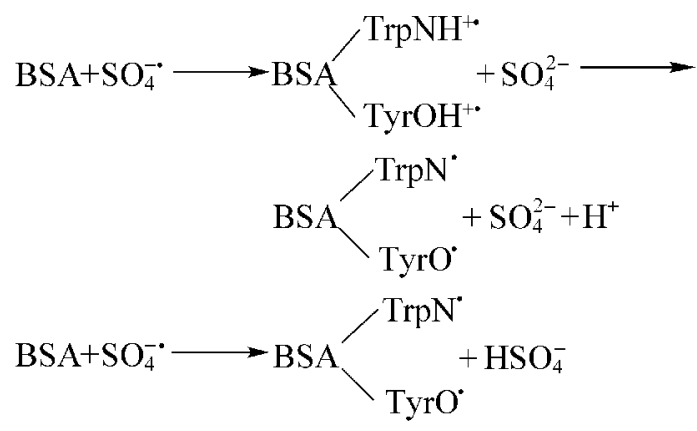

由于此二元体系中所产生的 $\mathrm{SO}_{4}^{-\bullet}$ 的浓度远远 大于其氧化所生成的氨基酸残基自由基的浓度, 故 保证二元体系中过硫酸钾的浓度不变, 在一定范围 内改变 BSA 的浓度, 分别计算 $460 \mathrm{~nm}$ 处 $\mathrm{SO}_{4}^{-\bullet}$ 的表 观衰减速率常数, 然后对BSA的浓度作图(见图5插 
图), 斜率即为 $\mathrm{BSA}$ 猝灭 $\mathrm{SO}_{4}^{-\bullet}$ 的反应速率常数, 求得 此值为 $1.51 \times 10^{10} \mathrm{~L} \cdot \mathrm{mol}^{-1} \cdot \mathrm{s}^{-1}$. 此值大于 $\mathrm{SO}_{4}^{-\bullet}$ 与氨基 酸反应的速率常数 ${ }^{[20]}$, 这是因为本实验所用的 BSA 的浓度是根据其分子量算得的摩尔浓度, 而非氨基 酸残基所表示的摩尔浓度.

\section{3 结 论}

利用纳秒级激光光解技术, 对 BSA 的紫外光损 伤和 $\mathrm{SO}_{4}^{-*}$ 氧化 BSA 的机理进行了系统的研究. 发 现在 $266 \mathrm{~nm}$ 激光作用下, BSA 中的 Trp 残基首先 以脱电子和发生分子内电子能级跃迁的形式受到破 坏, 进而通过分子内电子转移的形式氧化 Tyr 残基, 最终其光损伤以这两种氨基酸残基自由基的形式表 现出来. 而在 $\mathrm{SO}_{4}^{-\bullet}$ 氧化 $\mathrm{BSA}$ 的体系中, $\mathrm{SO}_{4}^{-\bullet}$ 可通过 与 BSA 中的 Trp 和 Tyr 残基发生电子转移而氧化 $\mathrm{BSA}$, 并且求得了这些氨基酸残基自由基的表观生 成速率常数, 获得了 $\mathrm{SO}_{4}^{-}$氧化 $\mathrm{BSA}$ 的反应速率常 数. 本研究为进一步理解 BSA 参与的某些生物体内 的氧化还原反应和探讨蛋白质的光损伤机理提供了 理论依据.

\section{References}

1 Deep, S.; Ahluwalia, J. C. Phys. Chem. Chem. Phys., 2001, 3: 4583

2 Vasilescu, M.; Angelescu, D.; Almgren, M.; Valstar, A. Langmuir, 1999, 15: 2635

3 Giancola, C.; de Sena, C.; Fessas, D.; Graziano, G.; Barone, G. Int J. Biol. Macromol., 1997, 20: 193

4 Saboury, A. A. J. Chem. Thermodyn., 2003, 35: 1975

5 Gelamo, E. L.; Itri, R.; Alonso, A.; Silva, J. V. D.; Tabak, M. J. Colloid Interface Sci., 2004, 277: 471

6 Hirayama, K.; Akashi, S.; Furuya, M.; Fukuhara, K. Biochem. Biophys. Res. Commun., 1990, 173: 639

7 Chu, G. S.; Song, Q. H.; Wang, Z. Y.; Ge, X. W.; Zhang, Z. C.; Wang, W. F.; Yao, S. D. Acta Phys. -Chim. Sin., 2000, 16(3): 232
[储高升, 宋钦华, 王忠义, 葛学武, 张志成, 王文峰, 姚思德. 物理 化学学报, 2000, 16(3): 232]

8 Sun, X. Y.; Zhang, C. J.; Wang, M.; Wang, S. L; Ni, Y. M.; Yao, S. D. Sci. China Ser. B, 2002, 32(4): 148 [孙晓宇, 张超杰, 王 玫, 汪世龙, 倪亚明, 姚思德. 中国科学 B 辑, 2002, 32(4): 148]

9 Zhao, P.; Wang, M.; Zhang, S. P.; Shao, S. C.; Sun, X. Y.; Yao, S. D.; Wang, S. L. Sci. China Ser. B, 2008, 38(7): 618 [赵 萍, 王 玫, 张淑萍, 邵思常, 孙晓宇, 姚思德, 汪世龙. 中国科学 B 辑, 2008, 38(7): 618]

10 Fang, H. J.; Dong, W. B.; Zhang, R. X.; Hou, H. Q. Acta Phys. Chim. Sin., 2006, 22(6): 761 [房豪杰, 董文博, 张仁熙, 侯惠奇. 物理化学学报, 2006, 22(6): 761]

11 Bensasson, R. V.; Land, E. J.; Truscott, T. G. Protein and component in excited states and free radicals in biology and medicine. Oxford: Oxford University Press, 1993: 103-142

12 Swagata, B.; Sharmistha, D. C.; Swagata, D.; Samita, B. J. Luminescence, 2008, 128: 437

13 Filipe, P.; Morlière, P.; Patterson, L. K.; Hug, G. L.; Mazière, J. C.; Freitas, J. P.; Fernandes, A.; Santus, R. Biochemistry, 2002, 41: 11057

14 Solar, S.; Getoff, N.; Surdhar, P. S.; Armstrong, D. A.; Singh, A. J. Phys. Chem., 1991, 95: 3639

15 Bent, D. V.; Hayon, E. J. Am. Chem. Soc., 1975, 97: 2612

16 Galian, R. E.; Pastor-Pérez, L.; Miranda, M. A.; Pérez-Prieto, J. Chem. Eur. J., 2005, 11: 3443

17 Deflippis, M. R.; Murthy, C. P.; Faraggi, M.; Klapper, M. H. Biochemistry, 1989, 28: 4847

18 Fu, H. Y.; Wu, G. Z.; Long, D. W.; Liu, Y. D.; Wang, W. F.; Yao, S. D. Acta Chim. Sin., 2006, 64(6): 483 [付海英, 吴国忠, 龙德 武, 刘耀东, 王文峰, 姚思德. 化学学报, 2006, 64(6): 483]

19 Zhu, H. P.; Zhang, Z. X.; Zhao, H. W.; Wang, W. F.; Yao, S. D. Sci. China Ser. B, 2006, 36(1): 76 [ 朱红平,张兆霞, 赵红卫, 王文峰, 姚思德. 中国科学 B 辑, 2006, 36(1): 76]

20 Wang, S. L.; Sun, X. Y.; Zhang, C. J.; Wang, M.; Li, W. Z.; Liu, S. H.; Ni, Y. M.; Yao, S. D. Sci. China Ser. B, 2002, 32(3): 278 [汪世龙, 孙晓宇, 张超杰, 王 玫, 李文哲, 刘仕姮, 倪亚明, 姚思德. 中国科学 B 辑, 2002, 32(3): 278] 